\title{
Coalescence Avalanches in Liquid Aluminum Foams
}

\author{
Marlen Paeplow ${ }^{1}$, Francisco García-Moreno ${ }^{1,2, *}$, Aaron Joseph Meagher ${ }^{2}$, Alexander Rack ${ }^{3}$ and \\ John Banhart ${ }^{1,2}$ \\ 1 Institute of Applied Materials, Energy Materials, Helmholtz Center Berlin, Hahn-Meitner-Platz 1, \\ 14109 Berlin, Germany; marlenpaeplow@gmail.com (M.P.); banhart@helmholtz-berlin.de (J.B.) \\ 2 Structure and Properties of Materials, Institute of Materials Science and Technology, Technical University \\ Berlin, Hardenbergstraße 36, 10623 Berlin, Germany; aaron.meagher@gmail.com \\ 3 X-ray Imaging Group ID-19, European Synchrotron Radiation Facility_ESRF, 71 Avenue de Martyrs, \\ 38000 Grenoble, France; alexander.rack@esrf.fr \\ * Correspondence: garcia-moreno@helmholtz-berlin.de; Tel.: +49-30-8062-42761
}

Received: 14 July 2017; Accepted: 1 August 2017; Published: 4 August 2017

\begin{abstract}
Coalescence is the rupture of a film between two adjacent bubbles in any type of liquid foam and has pronounced influence on the development of its macrostructure after solidification, mostly leading to larger pores and a wider size distribution. Foamable AlSi6Cu4 $+0.5 \mathrm{wt} \% \mathrm{TiH}_{2}$ precursors made following the powder metallurgical route were foamed in a gas-tight $\mathrm{X}$-ray transparent furnace under $500 \mathrm{kPa}$ ( 5 bar) pressure with subsequent pressure release. Coalescence was investigated by fast $X$-ray radioscopy at a frame rate of $1250 \mathrm{~Hz}$ and an effective pixel size of $9 \mu \mathrm{m}$ at the beamline ID-19, European Synchrotron Radiation Facility (ESRF), Grenoble, France. A self-developed program yielded the temporal and spatial detection of coalescence events in the acquired radioscopies. We observed the occurrence of film ruptures in a short temporal and spatial distance and we will refer to these phenomena as "avalanches".
\end{abstract}

Keywords: coalescence; avalanches; cascades; metal foam; film rupture; bubbles

\section{Introduction}

During metallic foam production, the liquid foam suffers morphological changes by effects such as drainage, flow, coarsening, and coalescence, which clearly influence the inner structure of the resulting solid foam [1]. In situ X-ray radioscopy is a powerful tool to image the evolution and formation of metal foams. An overview of the development of this method in the research field of metal foams within the last years is given by García-Moreno et al. [2]. Since the processes acting on the structure of a foam take place at different time scales, a careful choice of diverse time resolutions is required. A frame rate of about $1 \mathrm{~Hz}$ is sufficient for the investigation of, for instance, drainage or expansion. A high temporal resolution $(>10,000 \mathrm{~Hz})$, however, is mandatory for resolving coalescence, which starts with the rupture of the film and ends with the merging of the two adjacent bubbles, taking place in less than $1 \mathrm{~ms}$ [2-4]. The high frame rates of these radioscopies are achieved by the application of synchrotron radiation and high-speed cameras [5].

The study of film ruptures and bubble coalescence requires a fundamental analysis of the topological evolution of the foam structure to understand its stability. Coalescence of collapsing aqueous foams was simulated, measured, and analyzed quantitatively via optical and acoustic methods in the literature, whereby avalanches, also called cascades, were reported [6-14].

The focus of this study is on bubble coalescence in a liquid metal foam. The temporal and spatial occurrence of coalescence in metal foams was investigated in former studies with acquisition rates of up to $18 \mathrm{fps}$ [15-18]. We progressed this work and analyzed the occurrence of coalescence in liquid aluminum foams with capture rates of $1250 \mathrm{~Hz}$ at ID-19, European Synchrotron Radiation Facility 
(ESRF), Grenoble, France. The high frame rates enabled the observation of a new phenomenon: a fast local rearrangement of the liquid foam structure due to a large number of avalanche-like film ruptures, taking place in less than $1 \mathrm{~s}$.

\section{Materials and Methods}

\subsection{Production of the Foamable Precursors}

Foamable AlSi6Cu4 + $0.5 \mathrm{TiH}_{2}$ (all in wt \%) precursors were prepared by uniaxial hot-compaction of elemental powders ( $\mathrm{Al} D_{50}=63.9 \mu \mathrm{m}, \mathrm{Si} D_{50}=26.5 \mu \mathrm{m}$ and $\left.\mathrm{Cu} D_{50}=33.4 \mu \mathrm{m}\right)$ and the blowing agent $\mathrm{TiH}_{2}\left(D_{50}=14.1 \mu \mathrm{m}\right)$ following the powder metallurgical route [19] or by thixocasting [20]. After compaction the samples were cut to specified sizes. Table 1 gives an overview about process parameters and sample sizes.

Table 1. Process parameters and sample sizes about the manufacturing route of the used AlSi6Cu4 + 0.5 wt $\% \mathrm{TiH}_{2}$ precursors

\begin{tabular}{ccc}
\hline Process & Process Parameters & Sample Size in mm $^{\mathbf{3}}$ \\
\hline Uniaxial hot-compaction & $15 \mathrm{~min}, 400{ }^{\circ} \mathrm{C}, 300 \mathrm{MPa}$ & $10 \times 10 \times 3$ \\
Thixocasting & $640{ }^{\circ} \mathrm{C}, 6615 \mathrm{kN}, 0.3 \mathrm{~m} / \mathrm{s}$ & $20 \times 10 \times 2$ \\
\hline
\end{tabular}

\subsection{X-ray Radioscopy with Synchrotron Radiation}

The acquisition of radioscopies with high frame rates makes the use of synchrotron radiation mandatory. The quasi white spectrum of the wiggler at ID-19 provides a flux of $1.0 \times 10^{15}$ photons $/\left(\mathrm{mm}^{2} \cdot \mathrm{s}\right)$. To improve the contrast of the images, aluminum filters of different thicknesses are used. The transmitted beam hits a 300- $\mu \mathrm{m}$ thick YAG:Ce single crystal scintillator. Its ability to luminesce converts the $\mathrm{X}$-rays into visible light, which is detected by a CMOS-camera (PCO. 1200 h, PCO AG, Kelheim, Germany) combined with a lens system and mirror. It was possible to obtain radioscopies with a $1250 \mathrm{~Hz}$ repetition rate, $1280 \times 512$ pixel area, and $9 \mu \mathrm{m}$ effective pixel size (equalling a region of interest of $11.5 \mathrm{~mm} \times 4.6 \mathrm{~mm}$ ) with a total duration of $4.6 \mathrm{~s}$ limited by the camera RAM [3].

The self-made oven for foaming the precursors consists of a ceramic resistive heating plate surrounded by a hermetically sealed, X-ray transparent aluminum cylinder ( $40 \mathrm{~mm}$ diameter, $200 \mathrm{~mm}$ length). Through a gas inlet and outlet the pressure can be varied. During melting, the pressure was kept in Ar pressurised to $500 \mathrm{kPa}$ ( 5 bar). After the foaming temperature $\left(\sim 600^{\circ} \mathrm{C}\right)$ had been reached, the pressure was released, which enabled fast expansion in only a few seconds. Therefore, many coalescences happen in a short time range, which is preferred in this experiment because of the restricted time window available for image acquisition [21].

\subsection{Coalescence Detection in Radioscopies}

To analyze coalescences, it is crucial to measure their spatial and temporal occurrence. In recent years, programs have been developed to detect film ruptures in evolving metal foams automatically $[17,22]$. They are suitable for frame rates around $1 \mathrm{~Hz}$. Unfortunately, they are not useful for radioscopies with higher frame rates, since the applied frame rate of the radioscopies lies in the same temporal range as the rupture of a film. As the process of a single rupture would exist across several frames, current software solutions record the resulting rupture as a series of several independent ruptures at the same locus.

A self-written program in Python simplifies the temporal and spatial detection of coalescences in evolving metal foams by calculating intensity (grey scale value) different images between two subsequent images, allowing one to identify single rupture events over several images. In the actual version, the software is able to detect individual coalescence events, but not the size of the bubbles 
involved-i.e., the energy dissipation [12]—obtaining only the spatial distribution on the projected plane represented by the gravity center of the intensity difference. Figure 1 shows the process with two representative images of thixocast AlSi6Cu4 $+0.5 \mathrm{wt} \% \mathrm{TiH}_{2}$.

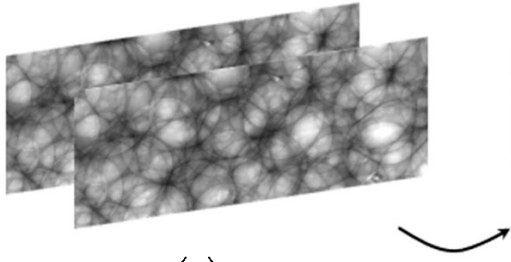

(a)

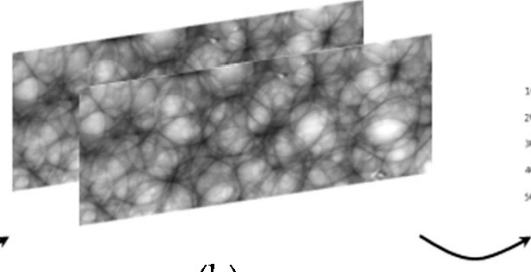

(b)

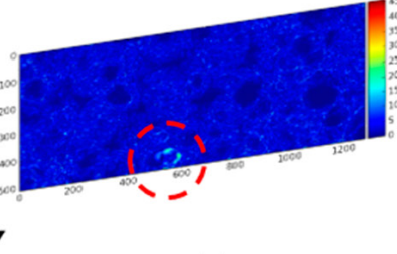

(c)

Figure 1. Functionality of the self-written Python program for temporal and spatial detection of ruptures. (a) Normalized, flat-field, and dark-field corrected images are (b) median filtered; (c) Afterwards the absolute intensity difference between two subsequent images is calculated. The resulting color-coded image highlights coalescence.

The first step (Figure 1a) is to normalize the flat-field and dark-field corrected images to produce a uniform brightness in the image sequences. Afterwards, we apply a median filter to reduce noise (Figure $1 \mathrm{~b}$ ). The last step is the calculation of the absolute intensity difference between each corresponding pixel in two subsequent images. The output image is color-coded, so that high difference values, like coalescence (the vanishing of a dark cell wall revealing a brighter background) are highlighted as marked with a red dashed circle in Figure 1c.

\section{Results and Discussion}

Figure 2a shows the cumulative coalescence count vs. time graph of a thixocast AlSi6Cu4 + $0.5 \mathrm{wt} \% \mathrm{TiH}_{2}$ sample obtained from the radioscopies. It contains the information about temporal occurrence of $\sim 135$ ruptures events during $\sim 4.5 \mathrm{~s}$. The total number of coalescence events depends on the number of bubbles, i.e., on the foaming stadium or foam age, heating ramp, expansion velocity, field of view, etc. The trigger of any coalescence event is the instability of the liquid films leading to rupture. The transition from a stable film to an instable one is often related to its thinning [23]. This can occur in liquid metals (i) during foam expansion due to film stretching, also called growth or expansion coalescence [24]; or (ii) due to foam collapse in the course of liquid drainage from the films, reaching a critical thickness beneath which the film breaks [15,24]. For metals foamed by the action of a blowing agent, rupture of films was found with the help of microgravity experiments to be also a consequence of pressure differences between bubbles due to an inhomogeneous distribution of the gas-supplying blowing agent particles [2].

The irregular slope of the graph-i.e., the temporal behavior of the cumulative coalescence count and therefore also of the coalescence rate-is explained by the uneven distribution of film ruptures in the period under consideration. The abrupt increases in slope (examples marked with black arrows in Figure 2a) are clearly seen in the radioscopies as avalanches of coalescence. The criteria to identify a series of coalescence events as an avalanche are their time and spatial correlation. A total of 3-50 events take place in our experiments in the range of 20-200 ms and are separated by less than one bubble diameter. The magnified area in Figure 2a shows one example for such an avalanche in detail. Each point is representing a single coalescence event. It was found that an avalanche takes only place if the triggering rupture corresponds to a large film, i.e., a large bubble is involved.

The irregular behavior of the cumulative coalescence count is in contrast to our previous results [25]. This can be attributed to the lack of time resolution of previous experiments. There, the accumulated number of film ruptures in $0.5 \mathrm{~Hz}$ radioscopies revealed a linear time dependence and no avalanches were detected. 


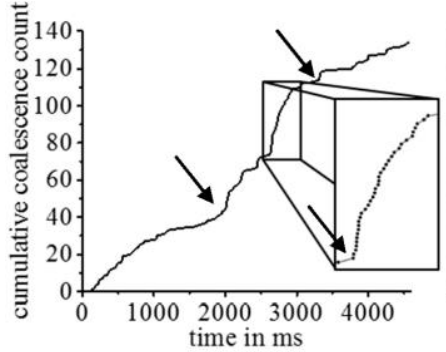

(a)

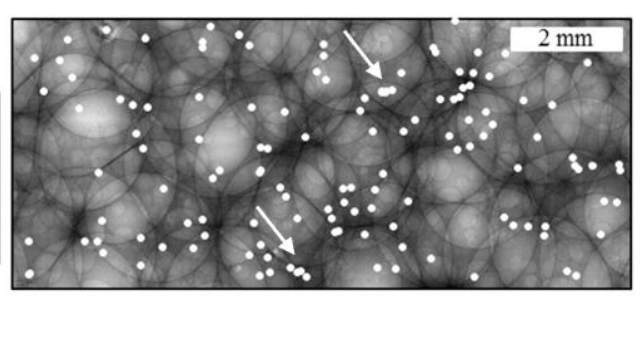

(b)

Figure 2. (a) Cumulative coalescence count vs. time with black arrows showing exemplary the starting point of three avalanches (one magnified as inset) and (b) coalescence map of a thixocast AlSi6Cu4 + $0.5 \mathrm{wt} \% \mathrm{TiH}_{2}$ liquid foam. White arrows indicate examples for close spatial distances between film ruptures. Each point in both images represents one single coalescence. A video of the whole sequence can be found online in the Supplementary Materials (Video S1).

Figure $2 \mathrm{~b}$ shows the result of the spatial rupture distribution-i.e., the coalescence map-overlapped with the first image of the radioscopy, which is displayed in the background of the coalescence map. White points represent each single coalescence event. In the center of large bubbles no coalescence was observed, as obviously there are no liquid films present. Film ruptures are mostly seen in plateau-borders, where smaller interstitial bubbles $(<250 \mu \mathrm{m})$ are located. Although they can be distributed in another way in the depth of the foam (they are projected in the X-ray direction), close spatial distance of the film ruptures is noticed in the 2D radioscopies and exemplary indicated with white arrows. The origin of the most lies in the avalanches, since the mechanical fluctuations of a breaking film and the subsequent merging of two bubbles can cause following coalescences due to topological rearrangements in the neighbourhood, and consequently a slope increase, in temporal behavior. This indicates a spatial and temporal correlation between coalescences corresponding to an avalanche. Several avalanches may appear at the same time but at different regions, although this behavior was not observed in our experiments, possibly due to the limited time and region studied.

To investigate the influence of the frame rate of image acquisition on the number of detected ruptures and the course of the graph, the time resolution of the radioscopy of a uniaxially hot-compacted AlSi6Cu4 sample was artificially reduced by a factor of 10 (to $125 \mathrm{~Hz}$ ) and a factor of 20 (to $62.5 \mathrm{~Hz}$ ). The results are presented in Figure 3. Compared with the original frame rate of $1250 \mathrm{~Hz}$, the cumulative coalescence with decreasing temporal resolution includes fewer events and shows a lower and smoother slope approaching a straight line. This is in good agreement with our previous results based on a 2500-times lower time resolution $(0.5 \mathrm{~Hz})[25]$ and corresponds to an integral value for the coalescence rate averaged over time.

The coalescence maps in Figure 3 as well as the cumulative coalescence count graph also show fewer accumulations at a decreasing sampling rate, which means that film ruptures with short spatial distances are detected less frequently. The local accumulation of events for high frame rates is higher, i.e., the spatial distribution for lower rates seems to be more uniform than for higher. The reason for this is that film ruptures that occur in a narrow spatial and temporal distance are easily misinterpreted as a single rupture when investigated at a lower frame rate and therefore avalanches are easily overlooked. Therefore, to resolve the kinetics of coalescence in detail, high frame rates are required, but with an acceptable contrast to detect individual events. For measurements of, e.g., the coalescence rate, a lower frame rate is sufficient. 


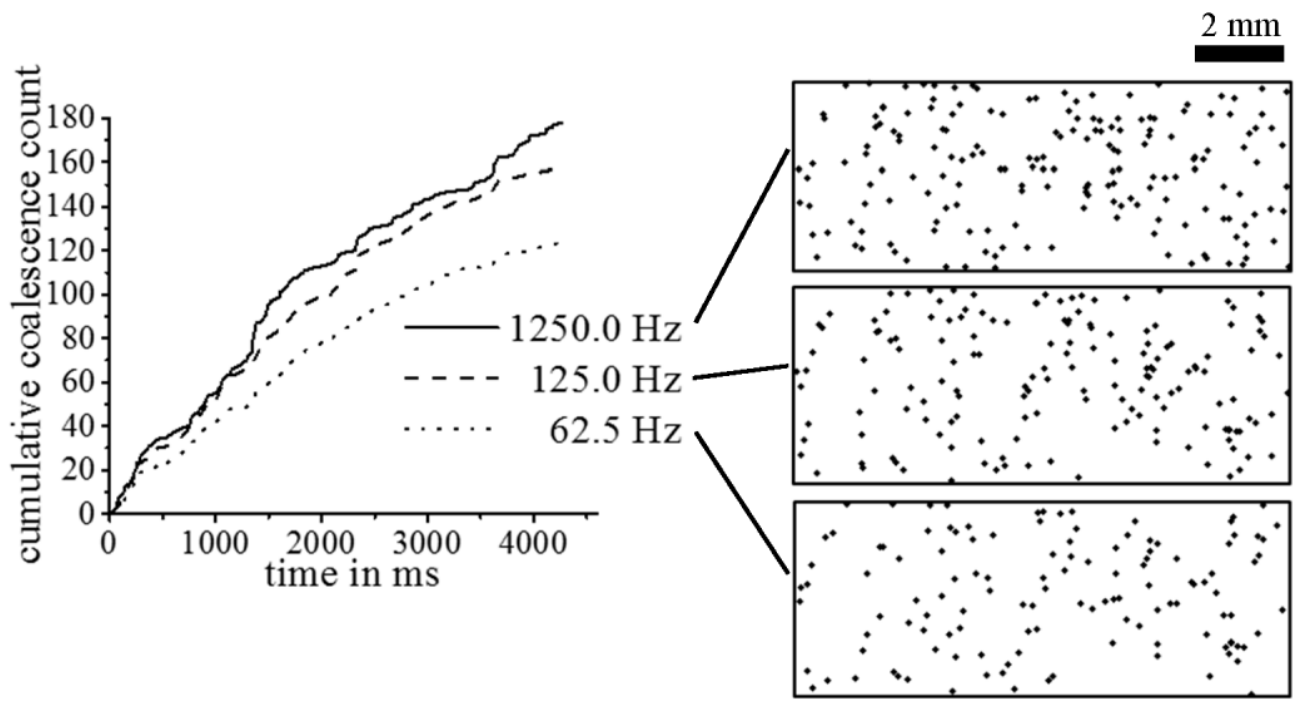

Figure 3. Cumulative coalescence count vs. time and coalescence maps of a uniaxially hot-compacted AlSi6Cu $4+0.5 \mathrm{wt} \% \mathrm{TiH}_{2}$ sample detected with image acquisition rates of $1250 \mathrm{~Hz}$ (compact line), $125 \mathrm{~Hz}$ (dashed line), $62.5 \mathrm{~Hz}$ (dotted line) with corresponding coalescence maps.

\section{Conclusions}

Coalescence avalanches in liquid aluminum foams were found, investigated, and described for the first time. The occurrence of subsequent film ruptures in a short spatial ( $<1$ bubble diameter) and temporal ( $<200 \mathrm{~ms}$ ) distance reveals fast local structural rearrangement in liquid AlSi6Cu4 $+0.5 \mathrm{wt} \%$ $\mathrm{TiH}_{2}$ foams produced in two different ways, namely thixocasting and uniaxial hot-compaction. The number of detected ruptures depends on the image acquisition rate: the higher the acquisition rate, the more ruptures are detectable. Therefore, coalescence avalanches can be only detected at high image sampling rates.

Supplementary Materials: The following are available online at www.mdpi.com/2075-4701/7/8/298/s1, Video S1: Coalescence of thixocast AlSi6Cu4.avi corresponding to Figure 2.

Acknowledgments: We thank Jörg Weise and Marco Haesche from IFAM Bremen for the thixocast samples. Research is supported by the European Space Agency ESA (MAP grant number AO-99-075:C14308/00/NL/SH) and German Research Foundation DFG (grants number BA 1170/35-1 and GA 1304/5-1).

Author Contributions: Francisco García-Moreno conceived and designed the experiments; Francisco García-Moreno and Alexander Rack performed the experiments; Marlen Paeplow and Aaron Joseph Meagher analyzed the data; Marlen Paeplow, Francisco García-Moreno, and John Banhart wrote the paper.

Conflicts of Interest: The authors declare no conflict of interest. The founding sponsors had no role in the design of the study; in the collection, analyses, or interpretation of data; in the writing of the manuscript, or in the decision to publish the results.

\section{References}

1. Banhart, J.; Baumgärtner, F.; Cox, S.J.; Kronberg, B.; Langevin, D.; Odenbach, S.; Weaire, D.; Wübben, T. Development of Advanced Foams under Microgravity. In Proceedings of the Microgravity Research and Applications in Physical Sciences and Biotechnology, Sorrento, Italy, 10-15 September 2000; pp. 589-596.

2. Garcia-Moreno, F.; Mukherjee, M.; Jiménez, C.; Rack, A.; Banhart, J. Metal Foaming Investigated by X-ray Radioscopy. Metals 2012, 2, 10-21. [CrossRef]

3. García-Moreno, F.; Rack, A.; Helfen, L.; Baumbach, T.; Zabler, S.; Babcsán, N.; Banhart, J.; Martin, T.; Ponchut, C.; Di Michiel, M. Fast Processes in Liquid Metal Foams Investigated by High-Speed Synchrotron X-ray Microradioscopy. Appl. Phys. Lett. 2008, 92, 134104. [CrossRef] 
4. Rack, A.; García-Moreno, F.; Baumbach, T.; Banhart, J. Synchrotron-Based Radioscopy Employing Spatio-Temporal Micro-Resolution for Studying Fast Phenomena in Liquid Metal Foams. J. Synchr. Radiat. 2009, 16, 432-434. [CrossRef] [PubMed]

5. Rack, A.; Garcia-Moreno, F.; Schmitt, C.; Betz, O.; Cecilia, A.; Ershov, A.; Rack, T.; Banhart, J.; Zabler, S. On the Possibilities of Hard X-ray Imaging with High Spatio-Temporal Resolution Using Polychromatic Synchrotron Radiation. J. X-ray Sci. Technol. 2010, 18, 429-441. [PubMed]

6. Burnett, G.D.; Chae, J.J.; Tam, W.Y.; De Almeida, R.M.; Tabor, M. Structure and Dynamics of Breaking Foams. Phys. Rev. E 1995, 51, 5788-5796. [CrossRef]

7. Hutzler, S.; Weaire, D.; Bolton, F. The Effects of Plateau Borders in the Two-Dimensional Soap Froth III. Further Results. Philos. Mag. B 1995, 71, 277-289. [CrossRef]

8. Okuzono, T.; Kawasaki, K. Intermittent Flow Behavior of Random Foams: A Computer Experiment on Foam Rheology. Phys. Rev. E 1995, 51, 1246-1253. [CrossRef]

9. Durian, D.J. Bubble-Scale Model of Foam Mechanics: Mmelting, Nonlinear Behavior, and Avalanches. Phys. Rev. E 1997, 55, 1739-1751. [CrossRef]

10. Hutzler, S.; Weaire, D. Foam Coarsening under Forced Drainage. Philos. Mag. Lett. 2000, 80, 419-425. [CrossRef]

11. Müller, W.; Di Meglio, J.M. Avalanches in Draining Foams. J. Phys. Condens. Matter 1999, 11, L209. [CrossRef]

12. Vandewalle, N.; Lentz, J.F.; Dorbolo, S.; Brisbois, F. Avalanches of Popping Bubbles in Collapsing Foams. Phys. Rev. Lett. 2001, 86, 179-182. [CrossRef] [PubMed]

13. Ritacco, H.; Kiefer, F.; Langevin, D. Lifetime of Bubble Rafts: Cooperativity and Avalanches. Phys. Rev. Lett. 2007, 98, 244501. [CrossRef] [PubMed]

14. Vandewalle, N.; Caps, H.; Dorbolo, S. Cascades of Popping Bubbles. Physica A 2002, 314, 320-324. [CrossRef]

15. Stanzick, H.; Wichmann, M.; Weise, J.; Helfen, L.; Baumbach, T.; Banhart, J. Process Control in Aluminum Foam Production Using Real-Time X-ray Radioscopy. Adv. Eng. Mater. 2002, 4, 814-823. [CrossRef]

16. Banhart, J.; Stanzick, H.; Helfen, L.; Baumbach, T. Metal Foam Evolution Studied by Synchrotron Radioscopy. Appl. Phys. Lett. 2001, 78, 1152-1154. [CrossRef]

17. García Moreno, F.; Fromme, M.; Banhart, J. Real-Time X-ray Radioscopy on Metallic Foams using a Compact Micro-Focus Source. Adv. Eng. Mater. 2004, 6, 416-420. [CrossRef]

18. Myagotin, A.; Ershov, A.; Helfen, L.; Verdejo, R.; Belyaev, A.; Baumbach, T. Coalescence Analysis for Evolving Foams via Optical Flow Computation on Projection Image Sequences. J. Synchr. Radiat. 2012, 19, 483-491. [CrossRef] [PubMed]

19. Banhart, J. Manufacture, Characterisation and Application of Cellular Metals and Metal Foams. Prog. Mater. Sci. 2001, 46, 559-632. [CrossRef]

20. Weise, J.; Stanzick, H.; Banhart, J. Semi-Solid Processing of Complex-Shaped Foamable Material. In Cellular Metals: Manufacture, Properties, Applications; Fleck, N.A., Banhart, J., Mortensen, A., Eds.; MIT: Berlin, Germany, 2003.

21. García-Moreno, F.; Babcsan, N.; Banhart, J. X-ray Radioscopy of Liquid Metalfoams: Influence of Heating Profile, Atmosphere and Pressure. Colloids Surf. A 2005, 263, 290-294. [CrossRef]

22. Myagotin, A.; Helfen, L.; Baumbach, T. Coalescence Measurements for Evolving Foams Monitored by Real-Time Projection Imaging. Meas. Sci. Technol. 2009, 20, 055703. [CrossRef]

23. Heim, K.; García-Moreno, F.; Kumar, G.V.; Rack, A.; Banhart, J. The Rupture of a Single Liquid Aluminum Alloy Film. Soft Matter 2014, 10, 4711-4716. [CrossRef] [PubMed]

24. Körner, C.; Arnold, M.; Singer, R.F. Metal Foam Stabilization by Oxide Network Particles. Mater. Sci. Eng. A 2005, 396, 28-40. [CrossRef]

25. Garcia-Moreno, F.; Solórzano, E.; Banhart, J. Kinetics of Coalescence in Liquid Aluminum Foams. Soft Matter 2011, 7, 9216-9223. [CrossRef]

(C) 2017 by the authors. Licensee MDPI, Basel, Switzerland. This article is an open access article distributed under the terms and conditions of the Creative Commons Attribution (CC BY) license (http:/ / creativecommons.org/licenses/by/4.0/). 